\title{
Correction to: SEOM clinical guidelines for endometrial cancer (2017)
}

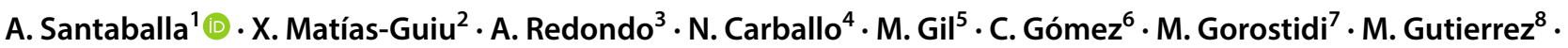 \\ A. Gónzalez-Martín ${ }^{9}$
}

Published online: 7 February 2018

(c) Federación de Sociedades Españolas de Oncología (FESEO) 2018

\section{Correction to: Clinical and Translational Oncology (2018) 20:29-37 https://doi.org/10.1007/s12094-017-1809-9}

In the original version of this article Fig. 1 was shown incorrectly. The correct Fig. 1 is shown here:

The original article can be found online at https://doi.org/10.1007/ s12094-017-1809-9.

\section{A. Santaballa \\ santaballa_ana@gva.es \\ X. Matías-Guiu \\ fjmatiasguiu.1leida.ics@gencat.cat
A. Redondo
aredondo12@gmail.com
N. Carballo
ncarballo@mdanderson.es
M. Gil
mgilmartin@iconcologia.net
C. Gómez
c_gomezraposo@hotmail.com

M. Gorostidi

mgorostidi@sego.es

M. Gutierrez maria.gutierreztoribio@ osakidetza.eus

A. Gónzalez-Martín agonzalezm@seom.org
1 Servicio de Oncología Médica, Hospital Universitari i Politècnic La Fe, Valencia, Spain

2 Servicio de Anatomía Patológica, Hospital Universitario de Bellvitge, Barcelona, Spain

3 Servicio de Oncología Médica, Hospital Universitario La Paz, Madrid, Spain

4 Servicio de Oncología Radioterápica, MD Anderson Cancer Center, Madrid, Spain

5 Servicio de Oncología Médica, Hospital Durán i Reynals, Barcelona, Spain

6 Servicio de Oncología Médica, Hospital Universitario Infanta Sofía, Madrid, Spain

7 Servicio de Ginecología, Hospital Universitario de Donostia, Donostia, Spain

8 Servicio de Oncología Médica, Hospital Unversitario de Araba, Vitoria, Spain

9 Servicio de Oncología Médica, Clínica, Universitaria de Navarra, Madrid, Spain 

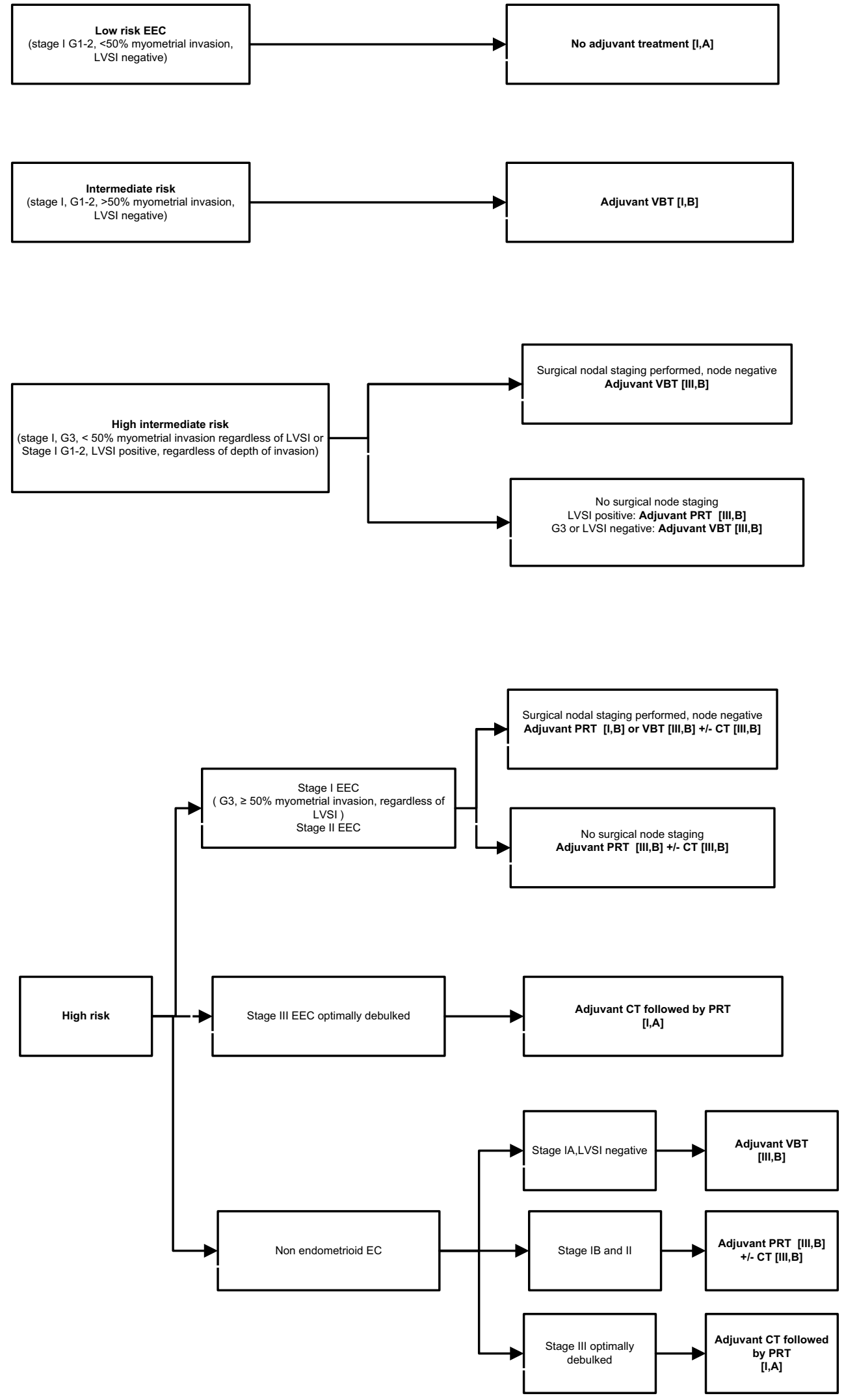

Fig. 1 Adjuvant treatment for endometrial cancer. LVSI lymphovascular invasion, $V B T$ vaginal brachytherapy, $C T$ chemotherapy, $P R T$ pelvic radiotherapy, $E E C$ endometrioid endometrial cancer, $E C$ endome- trial cancer. Modified from endometrial cancer algorithms that refers to Colombo et al. [7] 